Virginia Commonwealth University

vCU Scholars Compass

1999

\title{
The effect of carrier gas pressure on vapor phase nucleation experiments using a thermal diffusion cloud chamber
}

D. Kane

Virginia Commonwealth University

S. P. Fisenko

Virginia Commonwealth University

M. Rusyniak

Virginia Commonwealth University

M. S. El-Shall

Virginia Commonwealth University

Follow this and additional works at: http://scholarscompass.vcu.edu/chem_pubs

Part of the Chemistry Commons

Kane, D., Fisenko, S. P., Rusyniak, M., et al. The effect of carrier gas pressure on vapor phase nucleation experiments using a thermal diffusion cloud chamber. The Journal of Chemical Physics 111, 8496 (1999). Copyright (C) 1999 AIP Publishing LLC.

\section{Downloaded from}

http://scholarscompass.vcu.edu/chem_pubs/51

This Article is brought to you for free and open access by the Dept. of Chemistry at VCU Scholars Compass. It has been accepted for inclusion in Chemistry Publications by an authorized administrator of VCU Scholars Compass. For more information, please contact libcompass@vcu.edu. 


\title{
The effect of carrier gas pressure on vapor phase nucleation experiments using a thermal diffusion cloud chamber
}

\author{
D. Kane ${ }^{\text {a) }}$ S. P. Fisenko, ${ }^{\text {b) }}$ M. Rusyniak, and M. S. El-Shall ${ }^{\text {() }}$ \\ Department of Chemistry, Virginia Commonwealth University, Richmond, Virginia 23284-2006
}

(Received 4 June 1999; accepted 13 August 1999)

\begin{abstract}
Recent measurements of critical supersaturations for the vapor phase homogeneous nucleation of several substances using a diffusion cloud chamber technique exhibit a dependence on the pressure of the carrier gas used in the experiments. A model of droplet growth and motion in a diffusion cloud chamber, combined with the density and temperature profiles of the chamber is presented to explain the pressure dependent results. The model demonstrates that at higher carrier gas pressures the growth of the droplets is retarded and the optical scattering signal from the particles is reduced. It is concluded that the observed effect may not result from a pressure dependence of the nucleation rate, but from a pressure dependence of the droplet growth and motion. (C) 1999 American Institute of Physics. [S0021-9606(99)50542-4]
\end{abstract}

\section{INTRODUCTION}

Recently, we have reported that the critical supersaturation (the supersaturation, $S=P / P_{e}>1$, required for homogeneous nucleation at a rate of 1 drop $\mathrm{cm}^{-3} \mathrm{~s}^{-1}$, where $P$ is the actual pressure of the vapor at temperature $T$ and $P_{e}$ is the equilibrium vapor pressure at the same $T$ ) of glycerol measured in a He carrier gas, is dependent on the total pressure of the vapor-gas mixture. ${ }^{1}$ This dependence is qualitatively the same as the influence of the carrier gas pressure on the homogeneous nucleation of methanol, ethanol, 1-propanol, and 2-propanol reported in recent papers. ${ }^{2,3}$ The main conclusion from these studies is that increasing the carrier gas pressure, increases the critical supersaturation (i.e., decreases the nucleation rate). These results were obtained using an upward thermal diffusion cloud chamber (DCC). Other experiments in DCCs have also shown dependence of the nucleation rate on the carrier gas pressure. ${ }^{4-6}$ Chukanov et al. have observed a similar pressure effect in the nucleation of 1-pentanol, 1-butanol, 1-propanol, water and heavy water using $\mathrm{He}$ as a carrier gas. ${ }^{4}$ Katz et al. have found a weak effect due to the carrier gas in the nucleation of nonane in He and Ar. ${ }^{5,6}$

However, nucleation rate measurements carried out using other experimental techniques ${ }^{7-9}$ have not shown any detectable effect due to the nature or concentration of the carrier gas. Experiments with water in nitrogen as a carrier gas using supersonic nozzles did not exhibit any dependence on the carrier gas pressure. ${ }^{7,8}$ In expansion cloud chamber measurements using Ar as a carrier gas, no pressure effects were observed in the nucleation of 1-butanol, methanol, and water. ${ }^{9,10}$ An exception to these results is found in the recent work of Liujten et al. who studied the pressure effect in

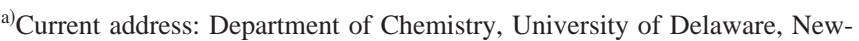
ark, Delaware 19716

b) Permanent address: A. V. Luikov Heat and Mass Transfer Institute, Academy of Sciences of Belarus, Minsk, Belarus.

${ }^{c}$ Author to whom correspondence should be addressed; electronic mail: selshall@hsc.vcu.edu
}

shock tube nucleation experiments. They found that the nucleation rate was pressure dependent when they calculated the supersaturation using real gas properties of the vapor-gas mixture. ${ }^{11}$

While the noncondensable carrier gas required in nucleation experiments is not considered within the classical nucleation theory (CNT) of Becker-Doring-Zeldovitch, there have been several recent theoretical treatments which attempt to clarify the role of the carrier gas in the nucleation process. ${ }^{12-22}$ The primary role of the carrier gas in removing the latent heat of formation of the new phase has been suggested since the early work of Kantrowitz. ${ }^{12}$ Feder, Russell, Lothe, and Pound ${ }^{13}$ have further developed this concept, and several papers have established that the role of the carrier gas is the removal of the latent heat from the growing clusters. ${ }^{14,17,18}$ At low concentrations of the carrier gas, effective removal of the excess energy is not possible. In this case the rate would decrease at lower carrier gas pressures, which is opposite to the effect observed in the DCC experiments. Kashchiev has derived a nucleation theory in which the nucleation rate and critical supersaturation are pressure dependent. $^{21}$ This theory suggests that the carrier gas pressure may increase or decrease the nucleation rate, depending on the nature of the carrier gas. This is in agreement with the work of Oxtoby and Laaksonen, who treated the homogeneous nucleation of a vapor in the presence of a carrier gas as a binary nucleation process. ${ }^{20}$ Using a kinetic approach, Bauer and Wilcox have also demonstrated a pressure dependence of the nucleation rate. ${ }^{15,16}$ The unanimous conclusion reached by these theoretical models is that the effect of the carrier gas pressure on the nucleation rate is too small to be observed experimentally. This conclusion appears reasonable since all liquids are practically incompressible at pressures less than the $4 \times 10^{6} \mathrm{~Pa}$ and the mean free path of vapor molecules at pressures typical of nucleation experiments is much larger than the critical cluster size. ${ }^{19}$ At pressures greater than $10^{6} \mathrm{~Pa}$ the mean free path of the vapor molecules is comparable to the critical cluster size and the nucle- 
ation kinetics change from free molecular to continuum or diffusion regime, and therefore nucleation kinetics may become dependent on the carrier gas pressure. ${ }^{19}$

However, a recent theoretical model developed by Novikov, Vasil'ev, and Reiss suggests that the impact parameter of a vapor molecule with the growing cluster is highly dependent on the concentration of the carrier gas. ${ }^{22}$ This is due to the screening of the attractive potential of the precritical cluster by the carrier gas. According to their results when the average intermolecular distance of the carrier gas is smaller than the critical cluster, a pressure effect may be observed. It is necessary to emphasize that the intermolecular distance being smaller than the critical cluster is a more limiting condition than the condition used by Fisenko, where the mean free path of the vapor is of the same order of magnitude as the cluster size. ${ }^{19}$ Recently another kinetic treatment of the pressure effect has been developed by Itkin,${ }^{23}$ in which he attempted to find a correlation between relaxation processes in clusters, nucleation rate and the carrier gas pressure.

Since pressure effects on homogeneous nucleation have most commonly been observed in DCC experiments, it is possible that these effects are mainly related to the nature of the experimental technique. Note that Katz and Fisk have recently shown that the pressure effect cannot be explained by the nonideality of the vapor-carrier gas mixture or the Poynting effects in the DCC experiments. ${ }^{6}$ However, one of the major limitations of the current nucleation experiments is the coupling between nucleation and growth. The nuclei, which typically have radii on the order of nanometers, are transient and once formed continue to grow. Nucleation experiments rely on light scattering from macroscopic droplets to detect the nucleation events. This permits the detection of particles no less than a few microns in diameter. Since the droplet must grow several orders of magnitude prior to detection, it is necessary to consider the possible role of droplet growth and motion on the experimental results.

In many nucleation experiments, such as expansion cloud chambers and shock tubes the process of droplet growth is decoupled from nucleation. However, in a DCC the processes of nucleation and droplet growth cannot be separated. It is normally assumed that each nucleation event results in the formation of a macroscopic droplet, which grows large enough to be detected. If the droplets do not grow to sufficient size before being removed from the vapor by reaching the top or bottom surface of the DCC, then an undercounting of the number of nucleation events may result. $^{24}$

In this paper, we present a model of growth and motion of droplets in a DCC to examine the role of carrier gas pressure on the size of droplets in the DCC. The structure of this paper is as follows. First the model is presented and its analytical predictions are discussed. Then the model and the effects of Mie scattering, in the limit of geometric optics, are used to explain the observed pressure dependence of the critical supersaturation of the homogeneous nucleation of glycerol. ${ }^{1}$ Application of the model to the homogeneous nucleation data of 1-propanol is also presented. Finally, the possibility of conducting nucleation measurements under mi- crogravity conditions in order to clarify the pressure effect on the droplets' growth is discussed.

\section{MATHEMATICAL MODEL}

For the purpose of the mathematical model, the DCC is considered to be two parallel liquid surfaces separated by a vertical distance $H$. The surfaces are at temperatures $T_{0}$ and $T_{1}$, where $T_{0}$, the temperature of the lower surface, is greater than $T_{1}$, the temperature of the upper surface. The volume between the two surfaces is maintained at a constant total pressure, $P_{T}$, by a light carrier gas, helium. The liquid on the lower surface of the chamber evaporates, diffuses vertically through the carrier gas and condenses on the upper surface. The steady state distributions of the density and temperature of the vapor as a function of height can be determined by solving the boundary value problem associated with the heat and mass flux in one dimension. ${ }^{25,26}$ The solution of these equations has been discussed in detail in Refs. 25 and 26, and many examples of thermodynamic profiles in cloud chambers are available in the literature..$^{1-3,5,6}$

By increasing the temperature difference between the two surfaces, $\Delta T=T_{0}-T_{1}$, the degree of supersaturation of the vapor, $S=P / P_{e}=n / n_{e}$ (where $n$ and $n_{e}$ are the actual number density of the vapor and equilibrium number density at a vapor-liquid interface, respectively) can be increased. At some temperature difference, nucleation will occur at a measurable rate. In a DCC, nucleation occurs in a small region around the maximum rate plane, which is defined here as the height at which the highest nucleation rate is predicted by the CNT.

Following nucleation, the droplet begins to grow and move within the chamber. Since the droplet radius changes by many orders of magnitude before it can be detected, it is necessary to consider two different regimes: free molecular and continuum, in which the growth and motion occur. The Knudsen numbers $\mathrm{Kn}_{\mathrm{v}}$ and $\mathrm{Kn}$ for cluster growth and droplet motion, as given by Eqs. (1) and (2), respectively, define these regimes:

$$
\begin{aligned}
& \mathrm{Kn}_{\mathrm{v}}=\lambda_{\mathrm{v}} / R, \\
& \mathrm{Kn}=\lambda / R,
\end{aligned}
$$

where $R$ is the cluster radius; $\lambda_{v}$ and $\lambda$ are the mean free paths of the vapor molecules and of the carrier gas, respectively. The mean free path of vapor molecules, $\lambda_{v}$, is given by

$$
\lambda_{\mathrm{v}}=\frac{1}{n_{g} a_{\mathrm{v}} \sqrt{1+M / m}},
$$

where $n_{g}$ is the number density of the carrier gas (usually helium or hydrogen), $a_{\mathrm{v}}$ is the transport cross-section of the vapor molecules with the background gas, $M$ and $m$ are the molecular weights of the vapor and carrier gas, respectively. The viscosity of the pure vapor has been used for the calculation of $a_{\mathrm{v}}$. Since the number density of the carrier gas is much greater than the vapor number density, in calculating the mean free path of carrier gas molecules it can be assumed 
TABLE I. Mean free path of the He carrier gas $(\lambda)$ and 1-propanol $\left(\lambda_{\vee}\right)$ vapor at different total pressures.

\begin{tabular}{ccc}
\hline \hline $\begin{array}{c}\text { Pressure } \\
(\mathrm{Pa})\end{array}$ & $\lambda(\mathrm{m})$ & $\lambda_{\mathrm{v}}(\mathrm{m})$ \\
\hline $1 \times 10^{5}$ & $7.8 \times 10^{-7}$ & $2.2 \times 10^{-7}$ \\
$5 \times 10^{5}$ & $1.6 \times 10^{-8}$ & $4.4 \times 10^{-8}$ \\
$1 \times 10^{6}$ & $2.2 \times 10^{-8}$ & $7.8 \times 10^{-8}$ \\
$5 \times 10^{6}$ & $4.4 \times 10^{-9}$ & $1.6 \times 10^{-9}$ \\
\hline
\end{tabular}

that collisions occur only between the background gas atoms. With this assumption the mean free path of the carrier gas, $\lambda$, is given by

$$
\lambda=\frac{1}{\sqrt{2} a n_{g}},
$$

where $a$ is the transport cross-section of the carrier gas. Using these Knudsen numbers it is possible to determine the regimes of droplet growth and motion. When $\mathrm{Kn}_{\mathrm{V}} \gg 1$, the droplet growth is in the free molecular regime. If $\mathrm{Kn}_{\mathrm{v}} \ll 1$, the growth is in the continuum or diffusion regime. ${ }^{19,23,24}$ Similarly, the motion of droplets is in free molecular regime if $K n \gg 1$, and if $K n \ll 1$, the motion of droplets is in the continuum regime. ${ }^{24}$

Table I lists the mean free paths of 1-propanol vapor and He carrier gas over the pressure range of the data from Ref. 2. Since the droplet must grow from $10^{-9} \mathrm{~m}$ to $>5 \times 10^{-6} \mathrm{~m}$ before it can be detected, it is apparent that growth and motion from the free molecular regime to the continuum regime must be considered. When the droplet number density is low enough that the density of the vapor is unaffected by the presence of the droplets, the equations of the motion and growth for the droplet can written as

$$
\begin{aligned}
& \frac{d\left(M_{d} \mathrm{v}\right)}{d t}=-M_{d} \bar{g}+F_{t}+F_{d}, \\
& \frac{d R(t)}{d t}=L(R, z)\left[n(z)-n_{e}(z)\right], \\
& \frac{d z(t)}{d t}=\mathrm{v}(R, t),
\end{aligned}
$$

where $z(t)$ is the position of droplet in the DCC at time $t$, $\mathrm{v}(R, t)$ is the velocity of the droplet, $M_{d}$ is the droplet mass, $\bar{g}$ is the acceleration of gravity, $F_{t}$ is the thermophoretic force, $F_{d}$ is the drag force on the droplet, $n(z)$ is the vapor density profile, $n_{e}(z)$ is the saturation vapor density profile, corresponding to the temperature $T(z)$, which is the temperature of the vapor-carrier gas mixture at height $z$ in cloud chamber. $L$ is the mass accommodation coefficient (the ratio of the mass transfer coefficient to the density of the condensed phase) expressed as a function of the droplet's radius and height in the chamber.

Expressions for the drag force, the thermophoretic force and the coefficient $L$, are well known from aerosol physics for the limiting conditions $\mathrm{Kn}_{\mathrm{V}} \ll 1$ and $\mathrm{Kn}_{\mathrm{v}} \gg 1$. Following
Vitovec et al., an interpolated expression for these properties with the correct asymptotic values, given by Eq. (8), has been used: ${ }^{24}$

$$
\frac{1}{X}=\frac{1}{X(F)}+\frac{1}{X(C)},
$$

where $X$ is a general property, $X(F)$ is the value of $X$ in the free molecular regime, and $X(C)$ is the value of $X$ in the continuum regime. In the model presented here the drag force in the free molecular regime is found from

$$
F_{d}(F)=-\frac{16}{3} R^{2} n_{g}\left(2 \pi m k_{B} T\right)^{1 / 2} \mathrm{v}
$$

and in a continuum medium the drag force is given by

$$
F_{d}(C)=-6 \pi \eta R \mathrm{v} .
$$

The thermophoretic force is given $b^{27}$

$$
F_{t}=\eta k_{B} \nabla T(z) \frac{F_{d}}{\mathrm{v}}
$$

and the coefficient $L$ is found from the two asymptotic expressions (12) and (13):

$$
\begin{aligned}
& L(F)=\frac{1}{\rho} \sqrt{\frac{M k_{B} T}{2 \pi},} \\
& L(C)=\frac{D M}{\rho R} .
\end{aligned}
$$

In Eqs. (9)-(13), $k_{B}$ is Boltzmann's constant, $\eta$ is the viscosity of the vapor-carrier gas mixture, $D$ is the diffusion coefficient of the vapor in the carrier gas and $\rho$ is the density of the liquid. The explicit expression for $L$ used in our calculations can be written as

$$
L=\frac{D}{\rho R}\left(\frac{1}{1+(D / R) \sqrt{\left(2 \pi M / k_{B} T\right)}}\right) .
$$

In a viscous medium the drag force will increase until it balances out the other forces. Therefore the droplet velocity is found from the condition that the sum of all forces on the droplet is equal to zero. This makes the droplet velocity dependent only on the radius of the droplet and its position. Equations (6) and (7) are then solved simultaneously, using a fourth-order Runge Kutta method, ${ }^{28}$ to obtain the droplet position and radius as a function of time. The initial conditions used in the solution of this system of equations are $t=0, R$ $=R_{0}$, and $Z=Z_{0}$, where $R_{0}$ and $Z_{0}$ are the initial radius and position of the droplet, respectively.

Because of the gradients in temperature and vapor density within the DCC, the free energy of cluster formation is dependent on the height in the chamber. ${ }^{29}$ Within the assumptions of CNT, the expression for the dimensionless free energy $\Delta \Phi(g, z) / k_{B} T(z)$ (where $g$ is the number of molecules in the cluster located at a reduced height $z$ in the chamber) is given by

$$
\frac{\Delta \Phi(g, z)}{k_{B} T(z)}=-g \ln (S(z))+\frac{4 \pi R(g)^{2} \sigma(T(z))}{k_{B} T(z)} .
$$




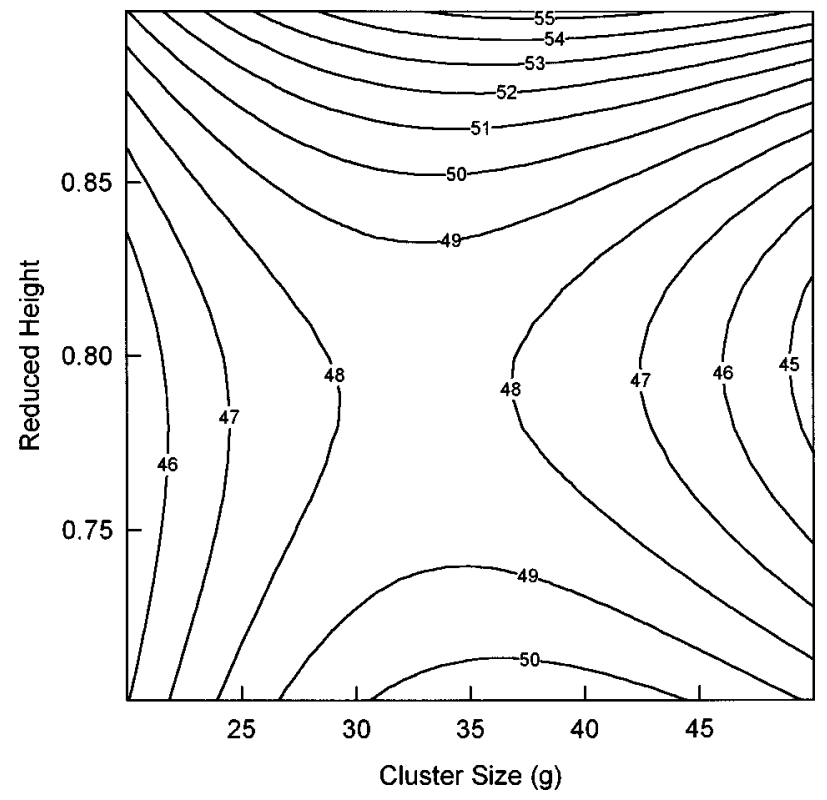

FIG. 1. Contour plot of the free energy surface for glycerol in a diffusion cloud chamber for the conditions of $T_{0}=437.5 \mathrm{~K}, T_{1}=347.9 \mathrm{~K}$, and $P_{\text {tot }}$ $=9.81 \mathrm{kPa}$.

In Eq. (15), $\sigma(T(z))$ is surface tension of the bulk liquid at temperature $T$ corresponding to the reduced height $z$ within the chamber. Figure 1 displays a sample of the contour plot of the free energy surface for supersaturated glycerol at the experimental conditions given in the caption of Fig. 1. The temperature and vapor density profiles used in the calculations are obtained using the steady-state solution of the diffusion and thermal conductivity equations, taking into account the dependence of the diffusion coefficient and thermal conductivity on temperature. The thermodynamic properties of the vapor and carrier gas used in the calculations are taken from Ref. 1. From Fig. 1 it is clear that the surface exhibits a saddle point whose position in the chamber can be located from the conditions:

$$
\frac{\partial}{\partial g}\left(\Delta \Phi\left(g_{c}, z_{c}\right) / k_{B} T\left(z_{c}\right)\right)=\frac{\partial}{\partial z}\left(\Delta \Phi\left(g_{c}, z_{c}\right) / k_{B} T\left(z_{c}\right)\right)=0 .
$$

The saddle point for supersaturated glycerol vapor (Fig. 1) at $T_{0}=437.5 \mathrm{~K}, T_{1}=347.9 \mathrm{~K}$, and $P_{\text {tot }}=9.8 \times 10^{3} \mathrm{~Pa}$ has the parameters $\Delta \Phi\left(g_{c}, z_{c}\right) / k_{B} T\left(z_{c}\right)=48.1, \quad g_{c}=32$ and $z_{c} / H=0.79$.

\section{ANALYTICAL PREDICTIONS OF THE MODEL}

In this section, we examine the predictions of the model analytically. At higher pressures most of the time the droplet growth and motion will be in the continuum regime, and the thermophoretic force can be neglected. The droplet velocity, $\mathrm{v}$, is then given by

$$
\mathrm{v}=-\frac{R^{2} \rho g}{3 \eta}
$$

Dividing Eq. (6) by Eq. (7), an expression for the radius of the droplet as a function of reduced chamber height, in the limit of the continuum regime, can be obtained as

$$
\frac{d R(z)}{d z}=\frac{3 D M \eta\left[n(z)-n_{e}(T(z=Z))\right]}{\rho^{2} R^{3} g} .
$$

By integrating this equation over the saddle point we obtain

$$
R^{4}(z)=\frac{12 M}{\rho^{2} g P} \int_{z_{c}}^{z} c(T(z)) \eta(T(z))\left(n(z)-n_{e}(z)\right) d z,
$$

where the temperature dependence of the viscosity and diffusion coefficient are taken into account and $D$ is expressed as $D=c(T) P^{-1}$ where $c$ is a temperature coefficient, since it is well known that $D \sim P^{-1}$. Due to the sharp maximum in the supersaturation profile, $S$, the integral in Eq. (19) can be approximately estimated as

$$
R^{4} \sim \frac{\eta\left(T\left(z_{m}\right)\right)\left(S_{m}-1\right) n_{e}\left(z_{m}\right) M\left(z-z_{c}\right)}{\rho^{2} g P},
$$

where $S_{m}$ is the maximum value of supersaturation in the nucleation zone, $n_{e}\left(z_{m}\right)$ is the number density of the saturated vapor, at the height of the saddle point. From expression (20) the dependencies of the droplet radius on the pressure and viscosity of the carrier gas are obvious. As supersaturation and saturation density profiles depend on the temperatures of the bottom plate, $T_{0}$, and the top plate, $T_{1}$, of the DCC, it is possible to increase the supersaturation, $S_{m}$, and simultaneously decrease the saturation density in such manner, that the final droplet radius decreases.

Since nucleation rates are measured by the scattering of light from the macroscopic droplets, which result from the nucleation events, it is necessary to include the effects of Mie scattering. When $2 \pi R / \lambda_{0} \gg 1$, where $\lambda_{0}$ is the wavelength of the light used for detection, the intensity of light scattered by the droplets, $I$, is proportional to $R^{2}$. Then from Eq. (20) it is evident that

$$
I \sim P^{-1 / 2} \text {. }
$$

For optical detection, the minimum radius of an object that can be detected depends on the particle index of refraction and the wavelength of the scattered light and characteristics of the detection system. If the final radius of the droplets is below the detection limit, then the observer must conclude that nucleation is not occurring.

\section{MODEL RESULTS}

In this section the model for droplet growth and motion is applied to examine the pressure effect observed in homogeneous nucleation rate measurements. In Fig. 2 the observed dependence of the critical supersaturation of glycerol on the total pressure is shown. Experimental details are available in Ref. 1. Here it is only necessary to mention that the conditions of the chamber necessary to produce a steady nucleation rate of 1-drop $\mathrm{cm}^{-3} \mathrm{~s}^{-1}$ were measured. The nucleation was observed by measuring the forward scattering of light as the droplets fall through a $\mathrm{He}-\mathrm{Ne}$ laser beam 


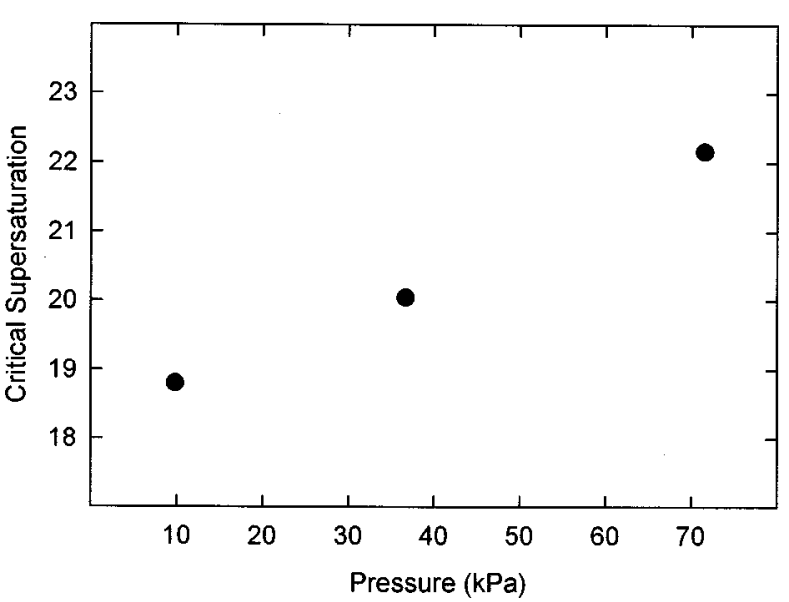

FIG. 2. Pressure dependence of the supersaturation required for the observation of droplets formed by homogeneous nucleation of glycerol at a rate of 1 drop $\mathrm{cm}^{-3} \mathrm{~s}^{-1}$.

directed horizontally across the lower half of the chamber. The forward-scattered light was collected by a PMT and a discriminator was used to distinguish droplet's signal from the background light. Care was taken to maintain identical conditions for counting, i.e., the same PMT amplification and discrimination levels in each experiment. In Fig. 2 a trend in increasing critical supersaturation at higher pressure is evident.

Figure 3 shows the results of the model simulation for glycerol droplet growth and motion in the DCC for the same experimental conditions of the data shown in Fig. 2. In Fig. 3 the droplet radius is given as a function of the reduced height in the chamber. It is clear that at a given reduced height, the droplets reach a smaller size as the pressure increases. Note that the effects of thermophoresis are apparent in the early stages of the cluster growth and are more prevalent at lower pressures. The initial upward motion of the particles appears to extend the time period for growth allowing the production of larger droplets. However, it is evident that as the pressure is increased, the droplet size is reduced. Over the range of pressures in Fig. 2 the droplet radius at a chamber-reduced

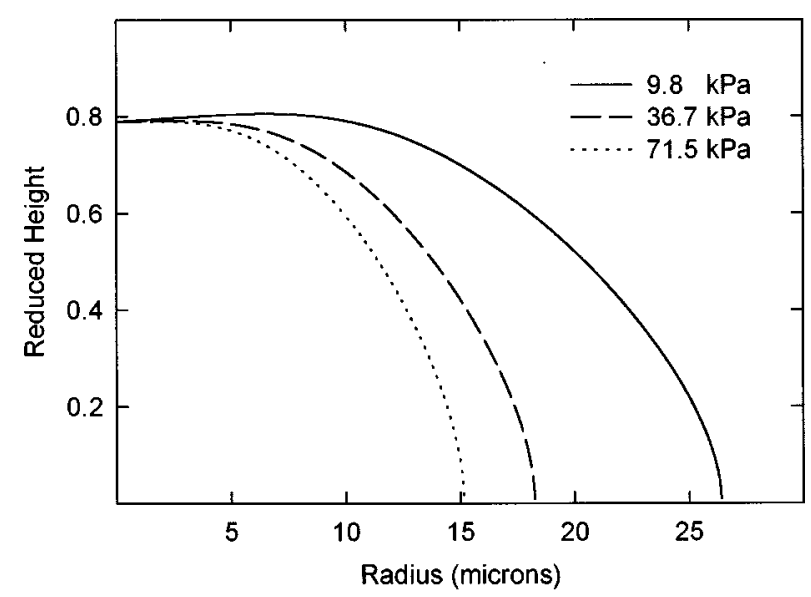

FIG. 3. Model simulation of the growth and motion of glycerol droplets across the diffusion cloud chamber at different total pressures, $T_{0}=437.5 \mathrm{~K}$, $T_{1}=347.9 \mathrm{~K}$.

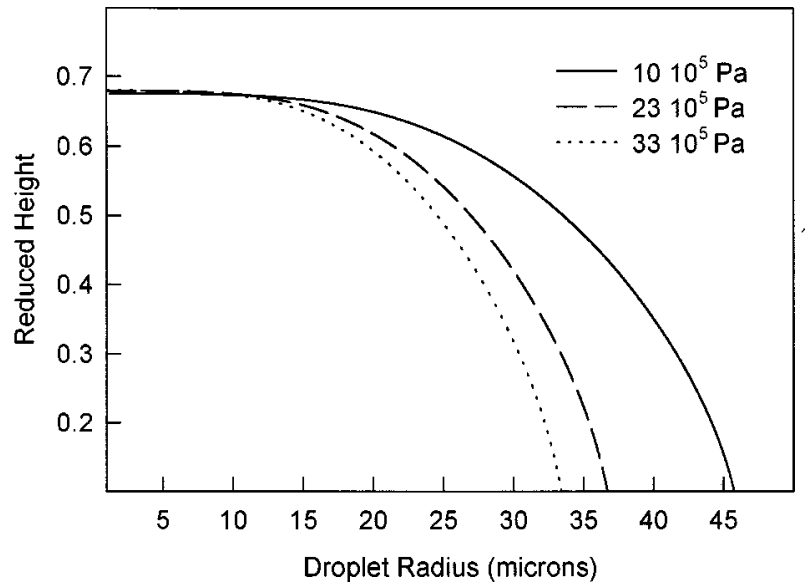

FIG. 4. Model simulation of the growth and motion of 1-propanol droplets across the diffusion cloud chamber at different total pressures, $T_{0}=393.8 \mathrm{~K}$, $T_{1}=308.0 \mathrm{~K}$.

height of 0.5 is decreased by about $29 \%$. This corresponds to a $50 \%$ reduction in the intensity of the scattered light or a $50 \%$ reduction in the height of the peak corresponding to scattered light signal from the droplet.

In Fig. 4 the droplet radius is given as a function of the reduced height in the chamber for the homogeneous nucleation of 1-propanol under the experimental conditions described in Ref. 2. The observed trend in Fig. 4 of smaller droplets at higher pressures is similar to that found for the nucleation of glycerol as shown in Fig. 3.

\section{DISCUSSION}

From the results of the model presented here, it is expected that the decrease in droplet radius at higher pressures resulted in an undercounting of the number of droplets and hence an underestimation of the nucleation rate. In these experiments the level of the discriminator must be set high enough that droplets falling on the edge of the $\mathrm{He}-\mathrm{Ne}$ laser beam or outside the viewing area of the PMT are not counted. As mentioned previously, in the glycerol experiment the discriminator circuit was not readjusted as the pressure of the carrier gas was increased. In this case the discriminator set at the lower pressures to count larger droplets was not properly adjusted to count the smaller droplets formed at higher pressures. This could lead to an apparent reduction of the nucleation rate. Although nucleation occurred and droplets were formed, the intensity of scattered light was below the detection limit and therefore the droplets were not accurately counted. In this case the temperature gradient was adjusted to increase the nucleation rate, until a sufficient rate was observed. This could result in an increase in the observed critical supersaturation.

It should be pointed out that the pressure effects might be more difficult or even impossible to correct under certain experimental arrangements. This is particularly true when the droplets are observed visually by the scattering of incoherent light against a white light field. It should be noted that according to Ref. 30 , it is only possible to visually observe particles with a radius greater than $25 \times 10^{-6} \mathrm{~m}$. The use of 
laser as a light source permits the observation of droplets with radii significantly smaller than 25 micron.

The simulations shown in Figs. 3 and 4 describe the growth and motion of droplets that form at the plane of the saddle point in the nucleation free energy surface. Similar calculations have shown that at a detection height of $z_{r}$ $=0.2$ the droplet size will vary by $\pm 5 \%$ for droplets originating over the width of the nucleation zone for the experimental conditions of Fig. 3. However, the difference in the radii of droplets formed at different heights in the chamber increases as the detection height is increased. If some of the droplets formed at different heights were too small to scatter significant amounts of light then an undercounting of the nucleation events would occur.

From the model presented here, it is evident that the diffusion coefficient and viscosity of the carrier gas influence the growth of droplets in the DCC experiments. Therefore, it can be concluded that the pressure and molecular properties of the carrier gas have significant effect on the dynamics of growth and motion of the droplets and can influence the apparent nucleation rate.

It should be noted that the analysis presented here was obtained for relatively low nucleation rates. In these calculations it was assumed that the growing droplets do not change the state of the vapor in the DCC. More complex effects arise in DCC nucleation experiments if the supersaturation is much higher than the critical supersaturation. In particular, oscillations in the nucleation rate can arise in the DCC. ${ }^{31}$ The "one droplet" approximation is not valid in this regime, because it is necessary to take into account the disturbance of the vapor density profile caused by the growing droplets. The primary impact takes place when the Knudsen number $\mathrm{Kn}_{\mathrm{v}}$ is smaller than one. The fulfillment of this condition depends on the pressure in the chamber. At higher pressure smaller droplets begin to deplete the supersaturated vapor and this will, in turn, affect the growth and motion of the droplets. The most interesting case occurs when the average distance between droplets is on the order of magnitude of the droplet's diameter.

\section{NUCLEATION STUDIES IN A MICROGRAVITY ENVIRONMENT}

If it is possible to increase the time required for the droplet to travel to the bottom surface of the chamber then it may be possible to study the role of the carrier gas pressure in the absence of the limiting factors affecting the droplet growth and motion. As mentioned above the primary forces that act to accelerate the droplet in the DCC are the gravitational force, the thermophoretic force and the drag force. Thermophoretic force acts in the direction of decreasing temperature, which is upward as we have defined the conditions of the chamber. The drag force always acts in a direction opposite to the velocity of the droplet. So the primary force responsible for the acceleration of the droplets toward the bottom surface of the chamber is the gravitational force.

Using the model presented above, it is possible to study the effects of reduced gravity on the growth and motion of droplets. The results of model calculations for the growth and motion of droplets under several different gravitational

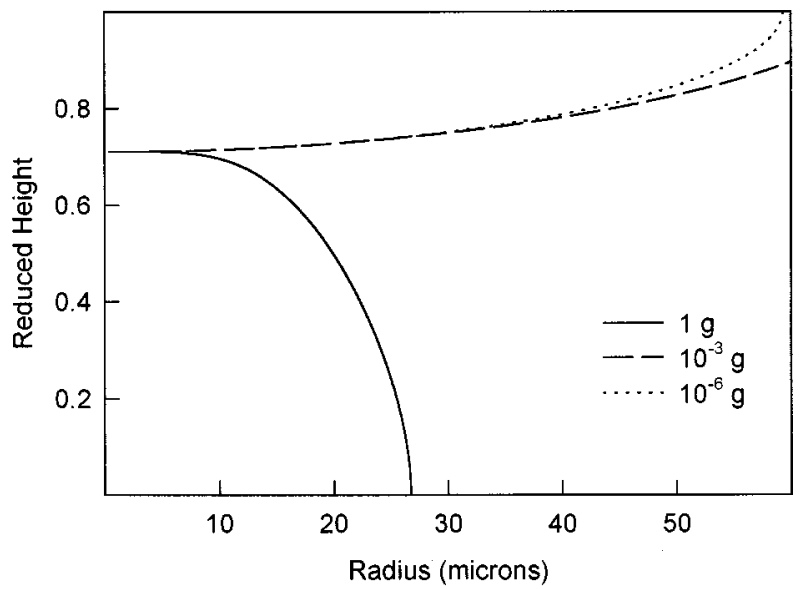

FIG. 5. Model simulation of the growth and motion of 1-propanol droplets for different gravity fields in the diffusion cloud chamber at $T_{0}=303 \mathrm{~K}$, $T_{1}=258 \mathrm{~K}$, and $P_{\text {tot }}=100 \mathrm{kPa}$.

forces are shown in Fig. 5. When the acceleration of gravity is reduced to $10^{-3} \mathrm{~g}$, the lifetime of the droplets in the chamber increases drastically to nearly 3 seconds. This allows enough time for the droplets to grow to greater than $30 \mathrm{mi}-$ crons in radius. At a gravity force of $10^{-6} \mathrm{~g}$, the droplet grows to greater than $0.5 \mathrm{~mm}$ and survives in the chamber for more than 5 seconds. Because of the low pressure of these simulations the droplets are removed to the upper surface of the chamber by the thermophoretic force. However, at higher pressures $(>1 \mathrm{~atm})$ this force will be negligible. This suggests that a long duration, high quality microgravity environment may be ideal for the study of the pressure effects of the carrier gas on the nucleation kinetics and the growth and motion of droplets.

\section{CONCLUSION}

Experimental studies to measure the effect of pressure on the nucleation rate must be aware of the effects of pressure on the growth and motion of the droplets, and account for these effects in the analysis of the data. Such experiments must not rely on visual observation of nucleation rates since the human eye is not as sensitive detector as a PMT. Only if steps are taken to eliminate the effects of droplet growth on the measured nucleation rate can the dependence of the nucleation kinetics on the carrier gas pressure be investigated.

\section{ACKNOWLEDGMENT}

The authors gratefully acknowledge support from the NASA Microgravity Materials Science Program (Grant No. NAG8-1484).

${ }^{1}$ D. Kane and M. S. El-Shall, J. Chem. Phys. 105, 7617 (1996).

${ }^{2}$ R. H. Heist, J. Ahmed, and M. Janjua, J. Phys. Chem. 98, 4443 (1994).

${ }^{3}$ R. H. Heist, J. Ahmed, and M. Janjua, J. Phys. Chem. 99, 375 (1995).

${ }^{4}$ V. N. Chukanov and B. A. Korobitsyn, Russ. J. Phys. Chem. 63, 1085 (1989).

${ }^{5}$ J. L. Katz, J. A. Fisk, and V. Chakarov, in Nucleation and Atmospheric Aerosols, edited by N. Fukuta and P. E. Wagner (Deepak, Hampton, VA, 1992), p. 11

${ }^{6}$ J. A. Fisk and J. L. Katz, J. Chem. Phys. 104, 8649 (1996). 
${ }^{7}$ G. Wilemski, B. E. Wyslouzil, M. Gauthier, and M. B. Frish, in Nucleation and Atmospheric Aerosols, edited by N. Fukuta and P. E. Wagner (Deepak, Hampton, VA, 1992), p. 23.

${ }^{8}$ B. E. Wyslouzil, G. Wilemski, M. G. Beals, and M. B. Frish, Phys. Fluids 6, 2845 (1994).

${ }^{9}$ P. E. Wagner, R. Strey, and Y. Viisanen, in Nucleation and Atmospheric Aerosols, edited by N. Fukuta and P. E. Wagner (Deepak, Hampton, VA, 1992), p. 27.

${ }^{10}$ Y. Viisanen, R. Strey, and H. Reiss, J. Chem. Phys. 99, 4680 (1993).

${ }^{11}$ C. C. Luijten, K. J. Bosschaart, and M. E. H. van Dongen, J. Chem. Phys. 106, 8116 (1997).

${ }^{12}$ A. Kantrowitz, J. Chem. Phys. 19, 1097 (1951).

${ }^{13}$ J. Feder, K. C. Russel, J. Lothe, and G. M. Pound, Adv. Phys. 15, 111 (1966).

${ }^{14}$ I. V. Skutova, S. P. Fisenko, and S. I. Shabunya, Sov. J. Chem. Phys. 3, 704 (1991).

${ }^{15}$ C. F. Wilcox and S. H. Bauer, J. Chem. Phys. 94, 8302 (1991).

${ }^{16}$ S. H. Bauer and C. F. Wilcox, Jr., J. Chem. Phys. 99, 4651 (1993).

${ }^{17}$ B. E. Wyslouzil and J. H. Seinfeld, J. Chem. Phys. 97, 2661 (1992).

${ }^{18}$ I. J. Ford, J. Aerosol Sci. 23, 447 (1992); J. C. Barett, C. F. Clement, and I. J. Ford, J. Phys. A 26, 529 (1993).
${ }^{19}$ S. P. Fisenko, J. Eng. Phys. Thermophys. 64, 470 (1993).

${ }^{20}$ D. W. Oxtoby and A. Laaksonen, J. Chem. Phys. 102, 6846 (1995).

${ }^{21}$ D. Kashchiev, J. Chem. Phys. 104, 8671 (1996).

${ }^{22}$ V. M. Novikov, O. V. Vasil'ev, and H. Reiss, Phys. Rev. E 55, 5743 (1997).

${ }^{23}$ A. L. Itkin, Chem. Phys. 238, 273 (1998).

${ }^{24}$ J. Vitovec, N. V. Pavlukevich, J. Smolik, and S. P. Fisenko, J. Eng. Phys. 56, 936 (1989).

${ }^{25}$ C. Becker, H. Reiss, and R. Heist, J. Chem. Phys. 68, 3585 (1978).

${ }^{26}$ J. Katz, J. Chem. Phys. 52, 4733 (1970).

${ }^{27}$ L. Talbot, R. K. Cheng, R. W. Scefer, and D. R. Willis, J. Fluid Mech. 101, 737 (1980).

${ }^{28}$ G. Arfken, Mathematical Methods for Physicists (Academic, Orlando, Florida, 1985), p. 492.

${ }^{29}$ D. Kane, S. P. Fisenko, and M. S. El-Shall, Chem. Phys. Lett. 277, 13 (1997).

${ }^{30}$ CRC Handbook of Chemistry and Physics (CRC, Boca Raton, Florida, 1986), p. F231.

${ }^{31}$ J. Brito and R. H. Heist, Chem. Eng. Commun. 15, 133 (1982). 